\title{
A Broadband High Gain, Noise-Canceling Balun LNA with 3-5 GHz UWB Receivers for Medical Applications
}

\author{
https://doi.org/10.3991/ijoe.v18i03.28009 \\ Thaar A. Kareem ${ }^{1,2(\bowtie)}$, Hatem Trabelsi ${ }^{1}$ \\ ${ }^{1}$ National Engineers School of Sfax, University of Sfax, Sfax, Tunisia \\ ${ }^{2}$ University of Misan, Misan, Iraq \\ thaar_kareem@uomisan.edu.iq
}

\begin{abstract}
The Ultra-Wideband Wireless Body Area Network (UWBWBAN) has been identified to provide an efficient, low-power, and improved wireless communication between sensor nodes worn by the human body to monitor physiological signals. The first part of the UWB receiver is a low noise amplifier (LNA). This article describes an upgrade to a sort of balun LNA that is entirely transistor-based and devoid of inductors for medical worn communication service. The balun LNA uses common gate and a common source configuration which cancels the noise generated by the common gate. This work uses the transistors in place of resistors to minimize the integrated circuit's area, as well as finding the best values for the dimensions of the transistor to minimize energy consumption, achieve a high gain and good linearity. This reduces the noise figure. The designed system utilizes the UWB frequency range of 3-5 GHz and a voltage supply of $1.8 \mathrm{~V}$. The designed balun LNA is able to achieve a peak gain of $25.5 \mathrm{~dB}$ and noise figure (NF) less than 3.2-3.5 dB using $180 \mu \mathrm{m}$ TSMC CMOS technology. The IIP3 is quite high at $2 \mathrm{dBm}$, whereas the IIP2 maximum is $21 \mathrm{dBm}$. The entire power consumption is less than $7.2 \mathrm{~mW}$.
\end{abstract}

Keywords-UWB, balun LNA, high gain, low NF, linearity, low power, CMOS

\section{$1 \quad$ Introduction}

A Wireless Body Area Network (WBAN) is a collection of sensors implanted or worn by the body. It allows information signals to be collected and communicated about a patient's health condition, such as heart rate and blood pressure, to a base station. This advancement aids in the prevention and diagnosis of issues while also preserving the patient's autonomy[1].

With the advancement in integrated circuits (IC) technology, biomedical applications with wireless communication have opened new paths to solve medical problems. The small-sized IC with low power consumption and transmission range can be embedded in the body and help in debugging the illness[2]. The modern circuits are inductorless to achieve the low cost and size[3]. The ultra wide band (UWB) technology is suitable for this. The UWB with a $7500 \mathrm{MHz}$ bandwidth from $3.1 \mathrm{GHz}$ to $10.6 \mathrm{GHz}$ 
[4][5] is approved by the Federal Communications Commission (FCC) in 2002. UWB has the advantages of low energy consumption and high transmission data rate. These advantages make the UWB a dominating technology [6]. UWB has grown in popularity due to its inexpensive cost, low transmission power, low power dissipation, high data rate, and resilience to multipath fading [7]. Due to hard attenuation of the transmitted signal, the signal at the received antenna is very weak. low noise amplifier (LNA) has to be used to amplify the received signal. The key objective of this article is to develop a very small, low-cost LNA with a high gain and good linearity LNA. Narrowband and wideband LNAs are readily available in the literature[8][9], but each has own drawbacks. Narrowband LNA makes the use of inductor circuits which make it bulky and energy hungry circuits. Though these have low noise figure but their integrated area is higher and requires technology with RF options to have inductors with high Q. On the other hand, wideband LNAs are complex in design. While RC wideband LNAs have a less complicated design, topologies exhibit high noise figures (NFs) [10]. Recently indcutorless LNAs are also proposed which unveils low NFs and require less on chip area [11][12].

The LNA design in this work is developed with the PMOS transistors as resistors [13]. As will be demonstrated, this strategy introduces a new degree of flexibility that may be exploited to optimize the LNA gain and, therefore, the circuit NF.

This document is structured in the following manner. In Section 2 and 3, we do an $\mathrm{AC}$ analysis to extract the equations for the balun LNA's basic CG and CS stages. We offer simulation findings for the typical LNA in Section 4. In Section 5, we compare the performance of this LNA to that of others previously published. Finally, Section 6 concludes the paper.

\section{Balun LNA analysis}

A critical option must be taken while designing a wideband LNA. A single-ended LNA doesn't need a balun to convert the single input to differential as balun reduces the NF substantially and higher losses[11]. It needs only the antenna and RF filters. However, the differential input gives lesser harmonic distortions and improved power supply with noise rejection.

The advantages of both single-ended and balun are used to investigate the LNA (see Figure 1) to design the simple and low-cost LNA. The load resistors have been eliminated in this design in favor of PMOS transistors and $\left(\mathrm{M}_{3}, \mathrm{M}_{4}\right)$ that operate in the triode region and are ideally approximated by a resistor between the drain and source.

$$
r_{d s}=1 / g_{d s}
$$

Where $g_{d s}$ is the channel conductance. To compare the suggested design with the LNA with load resistors, the initial design specifies the same resistance value for $r_{d s}$ as in the LNA with load resistors. 


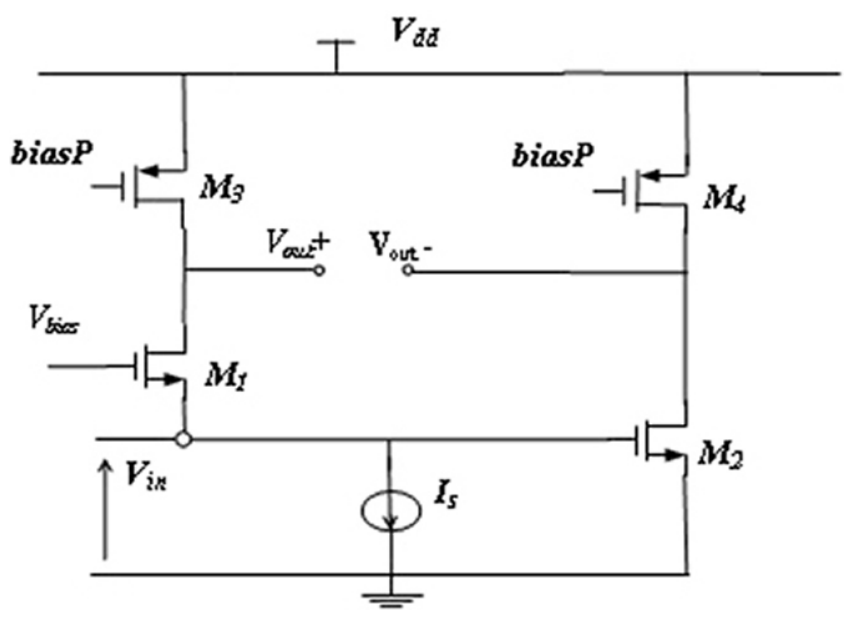

Fig. 1. Balun LNA

The suggested inductorless balun LNA circuit with combined common gate (CG) and common source (CS) configuration is illustrated in Figure 1. The single-ended input signal is amplified and transformed into differential output. The CG M1 transconductance $g_{m 1}$ governs the input impedance of this circuit. The noise at the input transistor $M_{1}$ is canceled when the gain of CS and CG are equal and opposite [14][15]. Additionally, distortion cancellation is possible, enabling the development of very linear LNAs. The impedance matching at the input is achievable if the CG-stage $Z_{\text {inCG }}$ input impedance equals to the source resistance $R_{s}$. We can modify the current in $M_{2}$ by adjusting the DC voltage (Vbias) at the gate of $\mathrm{M}_{1}$ in order to get an equal voltage gain at Vout+ and Vout-. The DC voltage (biasP) is utilized to alter the magnitude and NF of the LNA by adjusting the output conductance $\mathrm{g}_{\mathrm{ds} 3}$ and $\mathrm{g}_{\mathrm{d} 4}$ of $\mathrm{M}_{3}$ and $\mathrm{M}_{4}$, respectively.

\section{Small signal analysis}

Figures 2 and 3 represent equivalent small signal circuits of the common-gate stage and common-source stage, respectively. In the following equation $\boldsymbol{g}_{\boldsymbol{m}}$ and $\boldsymbol{g}_{\boldsymbol{m} \boldsymbol{b}}$ are the transistor's transconductance and body effect transconductance, respectively, $\boldsymbol{r}_{o}$ is the transistor's output resistance. The capacitance $\boldsymbol{C}_{g s}$ gate-source capacitance. $\boldsymbol{C}_{\boldsymbol{g} d}$ gatedrain capacitance, $\boldsymbol{C}_{s b}$ source-bulk capacitance and $\boldsymbol{C}_{d b}$ drain-bulk capacitance. $\boldsymbol{R}_{s}$ is the signal source resistance. We denote by $\boldsymbol{A}_{C G}$ and $\boldsymbol{A}_{C S}$ the voltage gain of common-gate circuit and common-source circuit, respectively. 


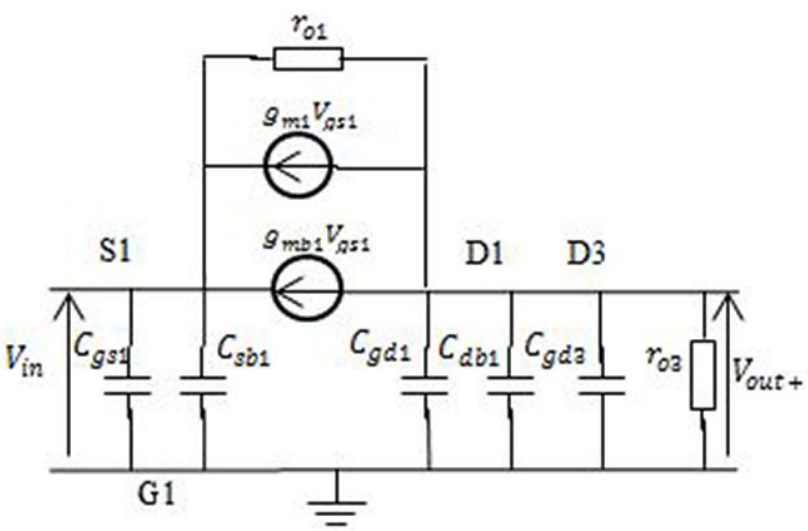

Fig. 2. Small signal circuits of the common-gate stage

\subsection{Small signal equivalent circuit common-gate and common-source stage}

In Figure 2 we denote by $\boldsymbol{Z}_{1}=\boldsymbol{r}_{\boldsymbol{o} 3} \| \frac{1}{j \boldsymbol{C}_{13} \boldsymbol{w}}$ and $\boldsymbol{C}_{13}=\boldsymbol{C}_{\boldsymbol{g} \boldsymbol{d} 1}+\boldsymbol{C}_{\boldsymbol{d b 1}}+\boldsymbol{C}_{\boldsymbol{g} \boldsymbol{d} 3}$. Here $\boldsymbol{V}_{\boldsymbol{i n}}=-\boldsymbol{V}_{\boldsymbol{g s}} ;$, when summing the currents on the output result in:

$$
\frac{V_{\text {out }}^{+}-V_{\text {in }}}{r_{\text {o1 }}}-g_{m 1} V_{\text {in }}+\frac{V_{\text {out }}^{+}}{Z_{1}}=0
$$

Now solving for $\frac{V_{\text {out }}^{+}}{V_{\text {in }}}$

$$
A_{C G}=\frac{V_{o u t}^{+}}{V_{\text {in }}}=\frac{r_{o 3}\left(1+\left(g_{m 1}+g_{m b 1}\right) r_{o 1}\right)}{r_{o 3}+r_{o 1}+j w C_{13} r_{o 1} r_{o 3}}
$$

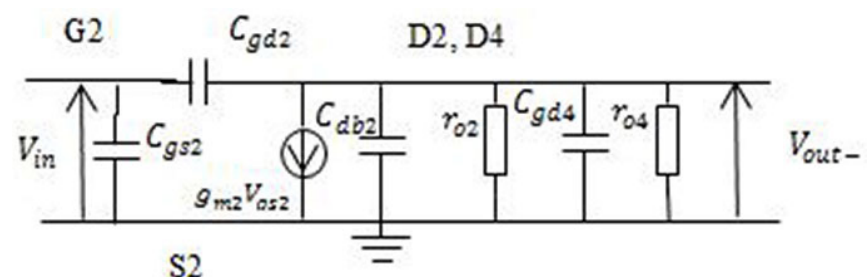

Fig. 3. Small signal circuits of the common-source stage

\subsection{Small signal equivalent circuit common-source stage}

For the common-source stage Figure 3 we denote by $\mathrm{R}_{\mathrm{o}}=r_{o 2} \| r_{o 3}$ and $C_{24}=C_{d b 2}+$ $C_{g d 4}$

Here $V_{i n}=V_{g s 2}$; summing the currents on the output result in: 


$$
\left(V_{\text {out }}^{-}-V_{\text {in }}\right) j w C_{g d 4}+g_{m 2} V_{\text {in }}+\frac{V_{\text {out }}^{-}}{R_{o} \| \frac{1}{j w\left(C_{24}\right)}}=0
$$

Solving for $\frac{V_{\text {out }}^{-}}{V_{\text {in }}}$ gives the voltage gain of common-source circuit:

$$
A_{C S}=\frac{V_{\text {out }}^{-}}{V_{\text {in }}}=-g_{m 2} R_{o}
$$

Capacitors $C_{g s 3}$ and $C_{g s 4}$ of transistors $\mathrm{M}_{3}$ and $\mathrm{M}_{4}$ don't affect the frequency behavior because both sides are connected to the AC ground. The differential gain of the LNA is given by:

$$
G=\frac{\left(V_{\text {out }}^{+}-V_{\text {out }}^{-}\right)}{V_{\text {in }}}=A_{C G}-A_{C S}
$$

If we neglect the effect of parasitic capacitance and $g_{m b}$, the common-gate voltage gain will be:

$$
A_{C G}=\frac{V_{o u t}^{+}}{V_{\text {in }}}=\frac{r_{o 3}\left(1+g_{m 1} r_{o 1}\right)}{r_{o 3}+r_{o 1}}
$$

and the common-source voltage gain will be:

$$
A_{C S}=\frac{V_{\text {out }}^{-}}{V_{\text {in }}}=-g_{m 2} R_{o}=-g_{m 2} \frac{r_{o 2} r_{o 4}}{r_{o 2}+r_{o 4}}
$$

In order to have a differential output circuit and cancels the output noise, the magnitude of the two gains should be equal. This is possible if we make; $r_{o 3}=r_{o 4}=r_{o P m o s}$; $r_{o 1}=r_{o 2}=r_{o N m o s} ;\left(g_{m 1}+g_{m b 1}\right) r_{o 1} \gg 1$ and neglecting the transistor body effect. Then $g_{m 1}=g_{m 2}$, so, it's important to fix the same length dimension $\mathrm{L}_{1}=\mathrm{L}_{2}=0.18 \mu m$ of the transistors M1 and M2. If we take in account those conditions the LNA gain will be expressed as in (9):

$$
A_{L N A}=\frac{\left(V_{\text {out }}^{+}-V_{\text {out }}^{-}\right)}{V_{\text {in }}}=A_{C G}-A_{C S}=2 g_{m 1} \frac{r_{\text {oNmos }} r_{\text {oPmos }}}{r_{\text {oNmos }}+r_{\text {oPmos }}}
$$

Indeed to attain the best performance a suitable scaling of the CS stage is required not only for a lower noise figure but also for lower distortion too. The nonlinearity of the transconductance $g_{m}$ and the output conductance $g_{d s}$ are the principal cause of this distortion besides the dependence of $g_{m}$ on the drain source bias voltage [14]. More a best balun -LNA linearity is obtained if the CS stage has the best linearity.

As the input impedance of the LNA is very significant for the overall receiver performance

$$
\begin{gathered}
Z_{\text {inLNA }}=Z_{\text {inCG }} \\
I_{\text {inCG }}=V_{\text {in }} j w\left(C_{g s 1}+C_{s b 1}\right)-\frac{V_{o u t}^{+}-V_{\text {in }}}{r_{o 1}}-\left(g_{m 1}+g_{m b 1}\right) * V_{g s 1}
\end{gathered}
$$


We denote $C_{2}=C_{g s 1}+C_{s b 1}$ and $C_{3}=C_{g d 1}+C_{d b 1}+C_{g d 3}$

$$
\begin{gathered}
-\frac{V_{o u t}^{+}-V_{\text {in }}}{r_{o 1}}-\left(g_{m 1}+g_{m b 1}\right) * V_{g s 1}=\frac{V_{o u t}^{+}}{r_{o 3}}+V_{o u t}^{+} * j w C_{3} \\
V_{o u t}^{+}=\left[\frac{r_{o 1+} r_{o 3}}{r_{o 1} r_{o 3}}+j w C_{3}\right]=\left(g_{m 1}+g_{m b 1}+\frac{1}{r_{o 1}}\right) V_{\text {in }} \\
I_{\text {inCG }}=j w C_{2} V_{\text {in }}-\frac{V_{o u t}^{+}=\frac{g_{m 1}+g_{m b 1}+\frac{1}{r_{o 1}}}{\frac{r_{o 1+} r_{o 3} r_{o 3}}{r_{o 1} r_{o 3}}+j w C_{3}} V_{\text {in }}+j w C_{3}}{r_{o n}}+\frac{1}{r_{o 1}} V_{\text {in }}+\frac{1}{r_{o 1}} V_{\text {in }}+\left(g_{m 1}+g_{m b 1}\right) V_{\text {in }} \\
\frac{I_{\text {inCG }}}{V_{\text {in }}}=j w C_{2}-\frac{g_{m 1}+g_{m b 1}+\frac{1}{r_{o 1}}}{\frac{r_{o 1+} r_{o 3}}{r_{o 1} r_{o 3}}+j w C_{3}}+\frac{1}{r_{o 1}}+\left(g_{m 1}+g_{m b 1}\right) \\
\frac{1}{Z_{i n C G}}=j w C_{2}-\frac{g_{m 1}+g_{m b 1}+\frac{1}{r_{o 1}}}{\frac{r_{o 1+} r_{o 3}}{r_{o 1} r_{o 3}}+j w C_{3}}+\frac{1}{r_{o 1}}+\left(g_{m 1}+g_{m b 1}\right)
\end{gathered}
$$

Neglecting the capacitance effects, we obtain

$$
\begin{gathered}
\frac{1}{Z_{i n C G}}=-\frac{g_{m 1}+g_{m b 1}+\frac{1}{r_{o 1}}}{\frac{r_{o 1+} r_{o 3}}{r_{o 1} r_{o 3}}}+\frac{1}{r_{o 1}}+\left(g_{m 1}+g_{m b 1}\right) \\
Z_{i n C G}=\frac{r_{o 1+} r_{o 3}}{r_{o 1}\left(g_{m 1}+g_{m b 1}\right)+1}
\end{gathered}
$$

\section{$4 \quad$ Simulation and result}

The analysis and simulation of the proposed wideband LNA is evaluated on the gain, noise figure and linearity. The simulation is done with the help of Advanced Design System (ADS) tool and TSMC RF $0.18 \mu \mathrm{m}$ CMOS process. 


\subsection{Gain, S11 and NF}

The $S_{11}$ plot in Figure 4 shows that the $S_{11}$ is less than $-15 \mathrm{~dB}$ for the frequency range of $3-5 \mathrm{GHz}$, which indicates that the designed LNA has good reflected coefficients in this frequency band. The range of the gain is equal 25.5-22.47 for the frequencies 3 to $5 \mathrm{GHz}$ which means we have a good performance for the gain, shown in Figure 5. The wideband LNA consumes $7.2 \mathrm{~mW}$ at a Vdd of $1.8 \mathrm{~V}$.

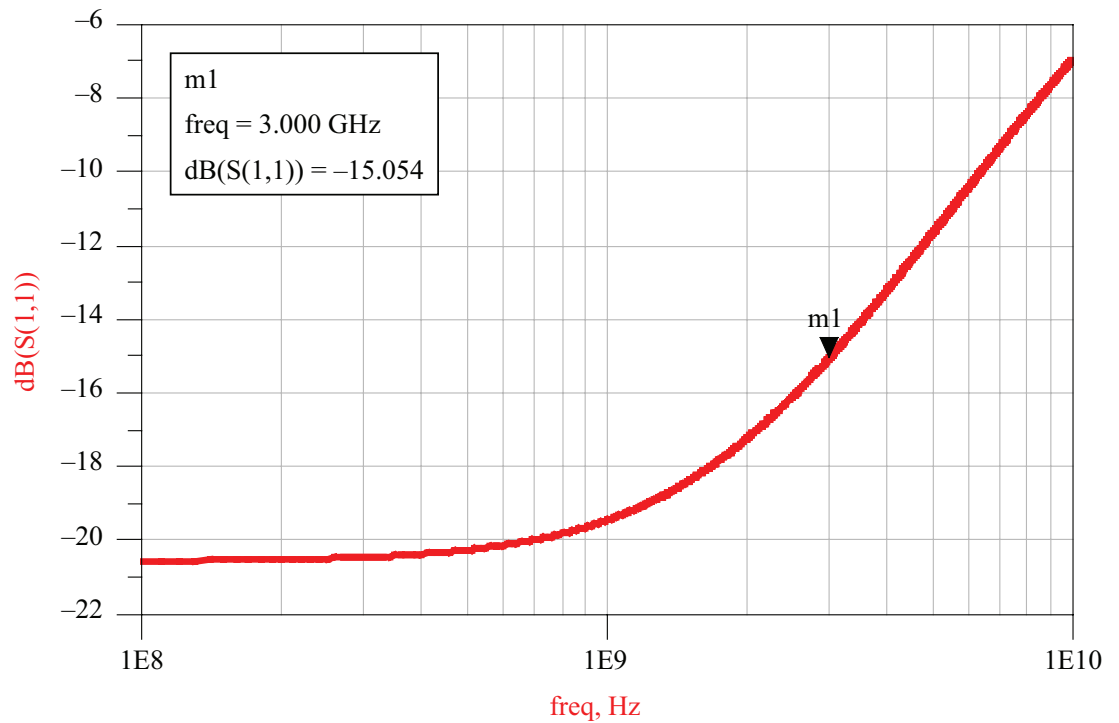

Fig. 4. S11 of the LNA

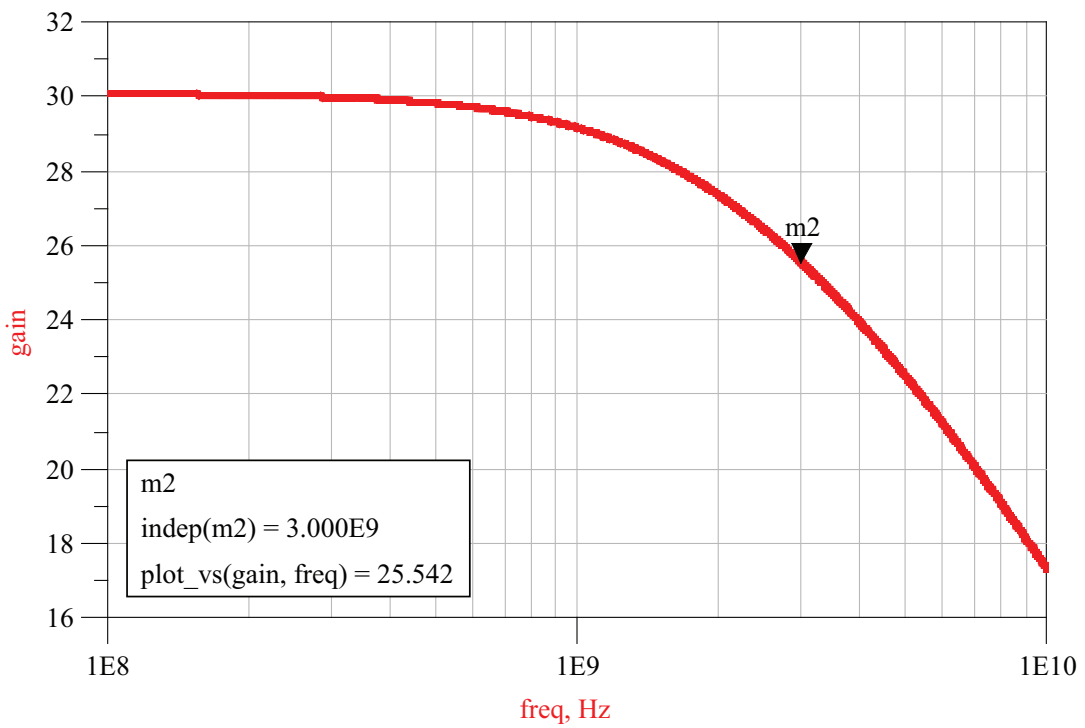

Fig. 5. Gain in $\mathrm{dB}$ of the LNA 
As shown in Figure 6, the Noise figure (NF) vary from $(3.2 \mathrm{~dB}$ to $3.5 \mathrm{~dB})$ for the frequencies 3 to $5 \mathrm{GHz}$.

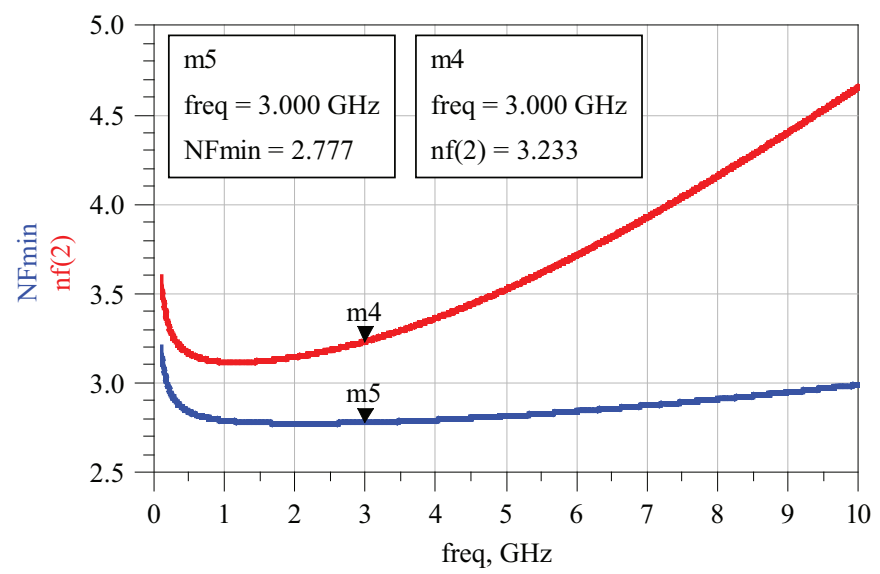

Fig. 6. NF of the LNA

\subsection{Linearity, IIP3, IIP2 and P-1dB}

The distortion performance is analyzed in the simulation for the second and third intermodulation intercept input IIP2 and IIP3, respectively and is depicted in Figures 7 and 8. An IIP3 of $2 \mathrm{dBm}$ and IIP2 $=21.018 \mathrm{dBm}$ are achieved and a $1 \mathrm{~dB}$ compression point $\mathrm{P}-1 \mathrm{~dB}($ in $\mathrm{dBm})=-14 \mathrm{dBm}$ as shown in Figure 9. Knowing that the UWB receiver receives a maximum power of $-41.3 \mathrm{dBm} / \mathrm{Mhz}$, the values found by simulation show that the LNA has an excellent linearity performance.

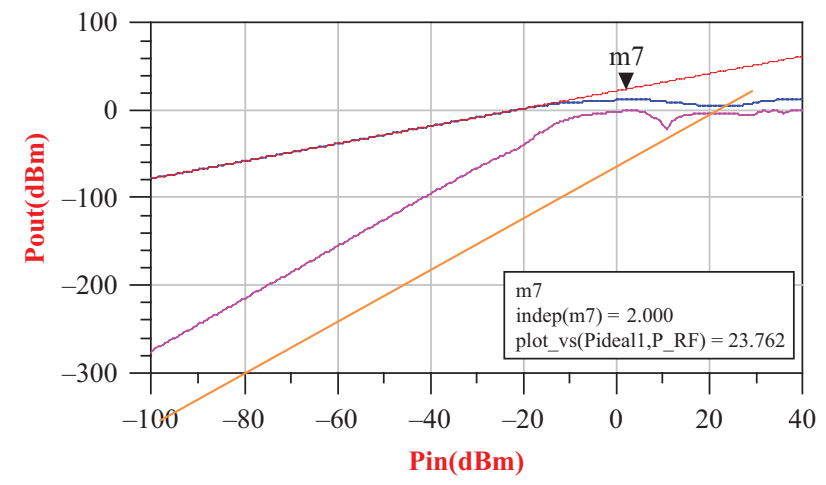

Fig. 7. IIP3 of the LNA 


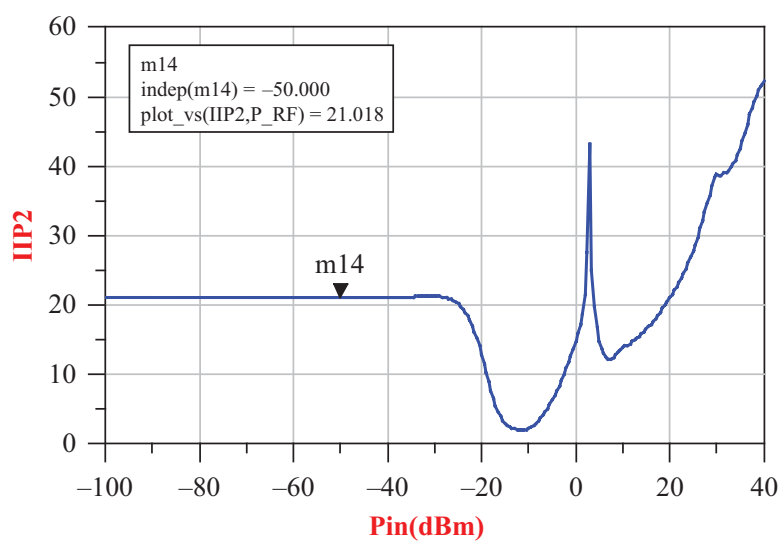

Fig. 8. IIP2 of the LNA

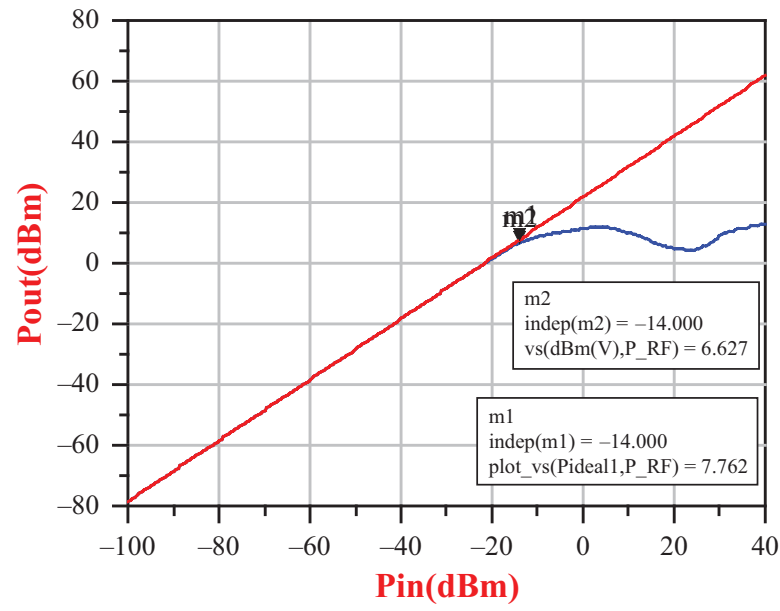

Fig. 9. $\mathrm{P}-1 \mathrm{~dB}($ in $\mathrm{dBm})$ of the LNA

\section{Comparison}

The results of the proposed circuit are compared with the state-of-the-art works, which devoid the inductor usage and are shown in Table 1. That the balun usage at the input with a mix of common source and common gate configuration has increased the gain, minimized the NF, reduces the IIP3 and improves the linearity. 
Table 1. LNA comparsion

\begin{tabular}{|l|c|c|c|c|c|c|c|c|}
\hline & $\begin{array}{c}\text { Freq. band } \\
(\mathbf{G H})\end{array}$ & $\begin{array}{c}\text { Tech. } \\
(\mathbf{n m})\end{array}$ & Gain (dB) & NF (dB) & $\begin{array}{c}\text { IIP2 } \\
(\mathbf{d B m})\end{array}$ & $\begin{array}{c}\text { IIP3 } \\
(\mathbf{d B m})\end{array}$ & $\begin{array}{c}\text { Power } \\
(\mathbf{m W})\end{array}$ & S11 \\
\hline$[6]$ & $3-5$ & 180 & 17 & $8-9$ & - & - & 26.6 & $<-10$ \\
\hline$[7]$ & $0.2-10$ & 130 & 12.2 & 3.2 & 6.2 & 0.7 & 4.8 & $<-10$ \\
\hline$[16]$ & $3-5$ & 180 & 16.8 & $2.6-3.1$ & & -3.47 & 35.1 & $<10$ \\
\hline$[17]$ & $0.2-5.2$ & 65 & $13-15$ & $<3.5$ & $>+20$ & $>0$ & 21 & $<-10$ \\
\hline$[18]$ & $0.8-6$ & 90 & $18-20$ & $<3.5$ & - & $>-3.5$ & 12.5 & $<-10$ \\
\hline This work & $3-5$ & 180 & $25.5-22.4$ & $3.2-3.5$ & 21.02 & 2 & 7.2 & $<-15$ \\
\hline
\end{tabular}

\section{Conclusion}

This article proposed a wideband LNA structure for high gain and low noise figure with excellent linearity performance. The proposed circuit can amplify the signals of frequencies between $3 \mathrm{GHz}$ and $5 \mathrm{GHz}$ with a gain ranging from $25.5 \mathrm{~dB}$ to $22 \mathrm{~dB}$ respectively, which shows good wideband performance. The design is based on the MOSFET only and uses the balun to convert the single-ended input to differential output with a mix of common gate and source stages. The analytical solution is also derived for gain and input matching. The validation of analytical solution is done through simulation in ADS software. The $180 \mathrm{~nm}$ CMOS technology simulation shows that, the NF is 3.2 to $3.5 \mathrm{~dB}$ for a power consumption of $7.2 \mathrm{~mW}$. The inductorless Balun-LNA is optimized in order to provide minimal NF, very good linearity, good gain, small chip area and low cost while consuming low power. This obeys to mostly chip constrains. In conclusion, the proposed inductorless Balun-LNA is the best candidate for the UWB transceiver used in the WBAN nodes.

\section{$7 \quad$ References}

[1] S. Benali and H. Trabelsi, "Analysis of device mismatches effect on the performance of UWB-Ring VCO," in 2020 17th International Multi-Conference on Systems, Signals \& Devices (SSD), 2020, pp. 814-818. https://doi.org/10.1109/SSD49366.2020.9364258

[2] Jihai Duan, Q. Hao2, Y. Zheng, B. Wei, W. Xu, and S. Xu, "Design of an incoherent IR-UWB receiver front-end in 180-nm CMOS Technology.” IEEE, p. 5, 2015. https://doi. org/10.1109/ISQED.2015.7085422

[3] D. Ben Issa, A. Kachouri, and M. Samet, "New design of 3.2-4.8 GHz generator for multibands architecture of UWB communication," Analog Integr. Circuits Signal Process., vol. 68, no. 1, pp. 9-20, 2011. https://doi.org/10.1007/s10470-011-9601-1

[4] J. H. C. Zhan and S. S. Taylor, "A 5GHz resistive-feedback CMOS LNA for low-cost multi-standard applications," Digest of Technical Papers-IEEE International Solid-State Circuits Conference, 2006. https://doi.org/10.1109/ISSCC.2006.1696111

[5] P. Sepidband and K. Entesari, "A CMOS wideband receiver resilient to out-of-band blockers using blocker detection and rejection," IEEE Trans. Microw. Theory Tech., vol. 66, no. 5, pp. 2340-2355, 2018. https://doi.org/10.1109/TMTT.2017.2783923 
[6] H. S. Madiha Hajri, Dalenda Ben Issa, "Low noise amplifier for MB-OFDM UWB receivers." IEEE, p. 4, 2016.

[7] J. G. I. Bastos, L. B. Oliveira, J. P. Oliveira and M. M. Silva, "Balun LNA with Continuosly Controlable Gain and with Noise and Distortion Cancellation.” IEEE, p. 4, 2012. https://doi. org/10.1109/ISCAS.2012.6271711

[8] D. Krstić, "RF microelectronics: Behzad Razavi: Prentice-Hall PTR, Upper Saddle River, NJ, 1998, hardcover, 335, ISBN 0-13-887571-5.” Elsevier, 1998.

[9] T. H. Lee, "The design of CMOS radio-frequency integrated circuits," Commun. Eng., vol. 2, no. 4, p. 47, 2004. https://doi.org/10.1017/CBO9780511817281

[10] F. Bruccoleri, E. A. M. Klumperink, and B. Nauta, "Wide-band CMOS low-noise amplifier exploiting thermal noise canceling," IEEE J. Solid-State Circuits, vol. 39, no. 2, pp. 275-282, 2004. https://doi.org/10.1109/JSSC.2003.821786

[11] I. Bastos, L. B. Oliveira, J. Goes, and M. Silva, "Analysis and design of a MOSFET-only wideband balun LNA," Int. J. Microelectron. Comput. Sci., vol. 1, no. 3, pp. 241-248, 2010.

[12] L. B. Oliveira, J. R. Fernandes, I. M. Filanovsky, C. J. M. Verhoeven, and M. M. Silva, Analysis and Design of Quadrature Oscillators. Springer Science \& Business Media, 2008. https://doi.org/10.1007/978-1-4020-8516-1

[13] T. Tille, J. Sauerbrey, M. Mauthe, and D. Schmitt-Landsiedel, "Design of low-voltage MOSFET-only/spl Sigma//spl Delta/modulators in standard digital CMOS technology," IEEE Trans. Circuits Syst. I Regul. Pap., vol. 51, no. 1, pp. 96-109, 2004. https://doi.org/10.1109/ TCSI.2003.821296

[14] Mouna Bettaieb, S. Benali, G. Bouzid, and HATEM Trabelsi, "Analysis and optimization of RF front-end for MICS band receiver." IEEE International Conference on Design \& Test of Integrated Micro \& Nano-Systems Analysis, p. 5, 2019. https://doi.org/10.1109/ DTSS.2019.8914911

[15] T. A. Vu, H. A. Hjortland, Ø. Næss, and T. S. Lande, “A 3-5 GHz IR-UWB receiver frontend for wireless sensor networks," in 2013 IEEE International Symposium on Circuits and Systems (ISCAS), 2013, pp. 2380-2383.

[16] S. X. Jihai Duan1, Qiangyu Hao2, Yu Zheng, Baolin Wei, Weilin Xu, "Design of an incoherent IR-UWB receiver front-end in 180-nm CMOS technology." IEEE, p. 5, 2015.

[17] S. C. Blaakmeer, E. A. M. Klumperink, D. M. W. Leenaerts, and B. Nauta, "Wideband balun-LNA with simultaneous output balancing, noise-canceling and distortion-canceling," IEEE J. Solid-State Circuits, vol. 43, no. 6, pp. 1341-1350, 2008. https://doi.org/10.1109/ JSSC.2008.922736

[18] R. Bagheri et al., "An 800-MHz-6-GHz software-defined wireless receiver in 90-nm CMOS," IEEE J. Solid-State Circuits, vol. 41, no. 12, pp. 2860-2876, 2006. https://doi. org/10.1109/JSSC.2006.884835

\section{Authors}

Thaar A. Kareem is a lecturer in Electric Engineering Department-College of Engineering, University of Misan, Iraq. He obtained BSC from university of Baghdad, Iraq and the master from EMU, north Cyprus. Now he is a $\mathrm{PhD}$ student in Systems Integration \& Emerging Energies Laboratory, Electrical Engineering Department, National Engineers School of Sfax, University of Sfax B.P. 1173, Sfax 3038, Tunisia, E-mail: thaar_kareem@uomisan.edu.iq. 
Hatem Trabelsi was born in Sfax, Tunisia in 1969. He received the engineering degree in electrical engineering from the National School of Engineers of Tunis in 1993, the aggregation degree in 2001, Ph.D. in electrical engineering in 2009 and HDR in electrical engineering from the National School of Engineers of Sfax in 2015. His research was mainly focused on Microelectronic circuit design and implementation of ultra low power transceivers for Wireless Sensor Network. He is a member of the research laboratory Systems Integration \& Emerging Energies Laboratory (SI2E), ENIS, where he is working in the field of analog and RF circuit design for ultra-wideband systems. His research interests are single-chip CMOS transceivers, RF front-ends, Ultra wideband analog circuit design. He is the author and co-author of several papers in the Microelectronic field. He is currently a Professor at the Electrical Engineering Department of the National School of Engineers of Sfax, University of Sfax B.P. 1173, Sfax 3038, Tunisia, E-mail: hatem.trabelsi@enis.tn.

Article submitted 2021-11-01. Resubmitted 2022-12-19. Final acceptance 2021-12-23. Final version published as submitted by the authors. 